\title{
Avaliação da Composição Nutricional, Teor Polifenólico e Atividade Antioxidante de Diferentes Espécies da Família Urticaceae
}

\author{
Michele do Nascimento ${ }^{l}$; Luana Minello'; Elizete Maria Pesamosca Facco'; Catia Santos Branco'; \\ Valdirene Camatti Sartori'; Gabriela Chilantil
}

csbrancl@ucs.br

1. Universidade de Caxias do Sul - UCS, RS.

Histórico do Artigo: 0 autor detém os direitos autorais deste artigo.

Recebido em: 07 de agosto de 2020 Aceito em: 15 de fevereiro de $2021 \quad$ Publicado em: 31 de agosto de 2021

Resumo: 0 interesse pelo estudo das "Plantas Alimentícias Não Convencionais- PANC's" vem crescendo, impulsionado, principalmente, pela tendência crescente de consumo pela população. Esse é o caso da família Urticaceae, a qual possui espécies descritas por possuírem propriedades nutritivas e que podem ser agregadas em produtos como um alimento funcional. Apesar dessas evidências, os estudos acerca da composição química e propriedades biológicas dessa família ainda são escassos. Nesse contexto, o objetivo do presente estudo foi avaliar a composição nutricional, a atividade antioxidante, o teor de fenólicos totais e compostos majoritários de quatro espécies da família Urticaceae (Boehmeria caudata Sw., Urera baccifera (L.) Gaudich. ex Wedd., Urtica circularis (Hicken) Sorarú e Urtica dioica (L.) coletadas no sul do Brasil. 0s resultados mostraram que a espécie U. baccifera foi a que apresentou o maior teor de umidade (11,02 \%) e de fibra alimentar (43,85\%). A espécie U. circularis apresentou o maior teor de minerais totais $(20,95 \%)$ e de proteína $(26,6 \%)$, enquanto a B. caudata obteve maior teor de carboidrato (7,64 \%). Com relação ao teor de fenólicos totais observou-se maior conteúdo para as espécies B. caudata e U. dioica $(274,92 \pm 6,05$ e $260,84 \pm 8,56 \mathrm{mg} \mathrm{GAE} / \mathrm{g}$, respectivamente), sendo a hesperidina o flavonoide majoritário, detectado na espécie U. baccifera $(116,49 \pm 0,94 \mu \mathrm{g} / \mathrm{mL})$. Tomados em conjunto, os resultados demonstram que as espécies da família Urticacea, tipicamente conhecidas pelas suas propriedades urticantes, podem ser consideradas boas fontes de nutrientes e de compostos bioativos com capacidade antioxidante.

Palavras-chave: Plantas alimentícias não convencionais, Urtiga, Composição centesimal, Polifenóis, Flavonoides.

\section{Evaluation of Nutritional Composition, Polyphenolic Content and Antioxidant Activity of Different Species of The Urticaceae Family}

\begin{abstract}
The interest in the study of "Unconventional Edible Plant" has been growing, driven mainly by the growing trend of consumption by the population. This is the case of the Urticaceae family that has species described by possessing nutritional properties, which can be added to products as a functional food. Despite this evidence, studies on the chemical composition and biological properties of this family are still scarce. In this context, the objective of the present study was to evaluate the nutritional composition, antioxidant activity, total phenolic content and major compounds of four species of the Urticaceae family (Boehmeria caudata Sw., Urera baccifera (L.) Gaudich. Ex Wedd., Urtica circularis (Hicken) Sorarú and Urtica dioica (L.) collected in southern Brazil The results showed that the U. baccifera species was the one with the highest moisture content (11.02\%) and dietary fiber (43.85 The species U. circularis had the highest content of total minerals $(20.95 \%)$ and protein $(26.6 \%)$, while B. caudata had the highest carbohydrate content (7.64\%). Regarding the total phenolic content, a higher content was observed for the species B. caudata and U. dioica (274.92 \pm 6.05 and $260.84 \pm 8.56 \mathrm{mg} \mathrm{GAE} \mathrm{/} \mathrm{g,} \mathrm{respectively),} \mathrm{with} \mathrm{hesperidin}$ being the flavonoid majority, detected in the species U. baccifera (116.49 $\pm 0.94 \mu \mathrm{g} / \mathrm{mL}$ ). Taken together, the results demonstrate that the species of the family Urticacea, typically known for their stinging properties, can be considered good sources of nutrients and bioactive compounds with antioxidant capacity.
\end{abstract}

Keywords: Unconventional edible plants, Stinging nettle, Centesimal composition, Polyphenols, Flavonoids. 


\section{Evaluación de la Composición Nutricional, Contenido Polifenólico y Actividad Antioxidante de Diferentes Especies de la Familia Urticaceae}

Resumen: El interés en el estudio de "Plantas alimentarias no convencionales - PANC" ha estado creciendo, impulsado principalmente por la tendencia creciente de consumo de la población. Este es el caso de la familia Urticaceae, que tiene especies descritas por tener propiedades nutricionales y que se pueden agregar a los productos como alimento funcional. A pesar de esta evidencia, los estudios sobre la composición química y las propiedades biológicas de esta familia aún son escasos. En este contexto, el objetivo del presente estudio fue evaluar la composición nutricional, la actividad antioxidante, el contenido fenólico total y los compuestos principales de cuatro especies de la familia Urticaceae (Boehmeria caudata Sw, Urera baccifera (L.) Gaudich. Ex Wedd., Urtica circularis (Hicken) Sorarú y Urtica dioica (L.) recolectadas en el sur de Brasil. Los resultados mostraron que la especie $U$. baccifera era la que tenía el mayor contenido de humedad (11.02\%) y fibra dietética (43.85 \%). La especie $U$. circularis tuvo el mayor contenido de minerales totales $(20.95 \%)$ y proteínas $(26.6 \%)$, y $B$. caudada tuvo el mayor contenido de carbohidratos $(7.64 \%)$. Con respecto al contenido fenólico total, se observó un mayor contenido para las especies B. caudata y U. dioica $(274.92 \pm 6.05$ y $260.84 \pm 8.56 \mathrm{mg} \mathrm{GAE} / \mathrm{g}$, respectivamente), siendo la flavonoide hesperidina, lo compuesto mayoritario, detectada en la especie U. baccifera $(116,49 \pm 0,94 \mu \mathrm{g} /$ $\mathrm{ml})$. En conjunto, los resultados demuestran que las especies de la familia Urticacea, tipicamente conocida por sus propiedades punzantes, puede considerarse una buena fuente de nutrientes y compuestos bioactivos con capacidad antioxidante.

Palabras clave: Plantas alimenticias no convencionales, Ortiga, Composición centesimal, Polifenoles, Flavonoides.

\section{INTRODUÇ̃̃o}

As plantas sempre fizeram parte da vida em sociedade. Historicamente, 0 uso delas têm sido coordenado por necessidades práticas, fatores culturais e conhecimentos tradicionais. Algumas espécies são amplamente distribuídas e seus usos são especialmente alimentícios (LEAL et al., 2018).

É o caso das plantas alimentícias não convencionais (PANC’s). Descritas por possuírem uma ou mais partes comestíveis, como raízes, tubérculos, bulbos, rizomas, talos, folhas, brotos, entre outras (KINUPP; BARROS, 2007), essas vem ganhando espaço em função da sua ampla distribuição e adaptação climática.

Segundo Sartori et al. (2020) tais plantas podem ser definidas como selvagens ou silvestres comestíveis, cuja importância reside no fato de que ainda pouco se conhece sobre os seus compostos bioativos e suas propriedades biológicas. Vistas como uma opção alternativa aos alimentos comumente consumidos em larga escala, as PANC's têm sido utilizadas para diversificar uma dieta monótona (CASEMIR0; VENDRAMIN, 2020). Além disso, evidências indicam sua importância e uso empírico na medicina tradicional (SARTORI et al., 2020).

A composição física, química e nutricional das PANC's depende da espécie, e está condicionada a diversos fatores intrínsecos e extrínsecos do metabolismo vegetal. Muitas 
dessas plantas têm sido relatadas por possuírem teores significativos de minerais, vitaminas, fibras e proteínas, além de apresentarem reconhecido efeito funcional e nutricional (OLIVEIRA et al., 2016). De modo geral, apresentam teores de nutrientes significativamente maiores do que as plantas domesticadas, além de serem ricas em fibras e em compostos antioxidantes, como os polifenóis (ALMEIDA; CORREA, 2012).

Também conhecidos como compostos fenólicos, os polifenóis são metabólitos secundários caracterizados pela presença de duas ou mais unidades de fenol. De acordo com sua estrutura química, esses podem ser divididos em flavonoides e não-flavonoides e apresentam diversas atividades biológicas, principalmente antioxidante (DEL RI0 et al., 2013; SOUZA et al., 2014).

A família Urticaceae é cosmopolita, ou seja, a distribuição das espécies é ampla, especialmente em regiões tropicais úmidas. No Brasil existem cerca de 12 gêneros e 80 espécies descritas para essa família.

Apresenta como particularidade a presença de pelos urticantes nas suas folhas e caules, que causam uma sensação de ardor devido à ação de compostos existentes no fluido destes pelos (tricomas), tais como a histamina, mas em processamento térmico os pelos urticantes e substâncias irritantes são destruídos (SILVA, 2017).

Embora algumas espécies sejam referidas como um alimento funcional (PEDERNEIRAS et al., 2011), pesquisas sobre suas propriedades nutricionais e biológicas ainda são escassas. Em estudo prévio, por exemplo, foi demonstrado que as folhas de urtiga da espécie $U$. baccifera contém substâncias antioxidantes, as quais exercem ação redutora através da varredura das espécies reativas de oxigênio (SILVA, 2017). A U. dioica, espécie mais estudada até o momento, é conhecida pelas suas propriedades antioxidantes e anti-inflamatórias (CARVALHO et al., 2017), tendo demonstrado efeitos neuroprotetores, cardioprotetores e antitumorais em estudos in vivo (para revisão, ver DHOUIBI et al., 2020).

Além dessas atividades, a $U$. dioica também é efetiva contra bactérias Gram-positivas e Gram-negativas (KREGIEL et al., 2018). Todas essas atividades têm sido atribuídas a matriz química da planta, especialmente rica em ácidos fenólicos e flavonoides (CARVALHO et al., 2017; KREGIEL et al., 2018; DHOUIBI et al., 2020).

Com base nessas evidências e considerando a importância das PANC's como uma alternativa que contribui para a sustentabilidade e soberania alimentar (SARTORI et al., 2020), novas pesquisas são necessárias. Nesse contexto, o presente estudo teve como objetivo avaliar a composição nutricional, o conteúdo fenólico, a identificação de compostos majoritários e a atividade antioxidante de quatro espécies da família Urticaceae da região sul do Brasil. 


\section{MATERIAL E MÉTODOS}

Realizou-se a coleta das espécies de B. caudata, U. baccifera, U. circularis e U. dioica, no município de Caxias do Sul e Nova Roma do Sul/ RS, no mês de setembro (2018) e março (2019) de acordo com a sazonalidade.

As amostras foram depositadas e identificadas no herbário da Universidade de Caxias do Sul sob a numeração 46748-B, 46747- U, 48681-U e 46749-U, respectivamente. As amostras (folhas) foram selecionadas aleatoriamente, secas em estufa com circulação de ar durante 24 horas $\left(45 \pm 5^{\circ} \mathrm{C}\right)$, em seguida foram trituradas para a obtenção de um pó e armazenado em local livre de iluminação e umidade até a realização das análises.

0 teor de umidade, cinzas, proteínas e lipídeos foram analisados de acordo com a metodologia do Instituto Adolfo Lutz (IAL, 2008). Inicialmente determinou-se o teor de umidade por estufa estabilizada a $105^{\circ} \mathrm{C}$, até a obtenção do peso constante, após incineração a $550^{\circ} \mathrm{C}$ em mufla para análise de cinzas.

A determinação de proteínas foi realizada através do método Kjeldahl, utilizando o fator de conversão 6,25 (N x 6,25), e a extração com solventes específicos. Os lipídeos foram extraídos sem aquecimento, e os solventes utilizados foram: clorofórmio, metanol e água. A determinação de fibras foi determinada seguindo o método enzimático-gravimétrico proposto pelo método AOAC (2011).

Os resultados da análise centesimal foram expressos em g/100 g. A análise de carboidratos totais foi determinada por diferença, sendo $100-(\mathrm{g}$ umidade $+\mathrm{g}$ proteína $+\mathrm{g}$ lipídeo + $\mathrm{g}$ cinza). Para determinação do valor calórico foram utilizados os resultados em gramas obtidos para carboidratos, proteínas e lipídeos, somados aos fatores de conversão específicos (Carboidratos - 4kcal/g; Proteínas - 4kcal/g; Lipídeos -9kcal/g), de acordo com A0AC (2011).

Para análise de compostos fenólicos e atividade antioxidante, o pó das folhas foi utilizado para o preparo de um extrato aquoso ( $1 \%$, p/v) obtido por decocção $\left(100{ }^{\circ} \mathrm{C} ; 15\right.$ min.). As suspensões resultantes foram centrifugadas a $3220 \mathrm{~g}$ a $4^{\circ} \mathrm{C}$ durante 15 minutos e o sobrenadante foi utilizado nas análises. A análise do teor de compostos fenólicos foi realizada pelo método espectrofotométrico de Folin-Ciocalteau (SINGLETON; ROSSI, 1965). 
Os resultados foram expressos em mg GAE/100 g. A identificação de compostos fenólicos foi realizada através da Cromatografia Líquida de Alta Eficiência (CLAE), seguindo a metodologia descrita por Morelli (2011).

Os compostos majoritários foram identificados de acordo com sua ordem de diluição e por comparação de seu tempo de retenção com aqueles de seus padrões puros. A quantificação foi realizada pelo método de padronização externa, através da correlação da área (mAU*s) do pico do composto à curva padrão realizada com cada padrão avaliado (ácido gálico, epigalocatequina, catequina, epicatequina, epigalocatequina galato, rutina, ácido ferúlico, naringina, hesperidina, miricetina, resveratrol, quercetina, apigenina e canferol). Os resultados foram expressos em $\mu \mathrm{g} / \mathrm{mL}$ de extrato.

A avaliação da atividade antioxidante se deu pelo método de varredura do radical DPPH (1,1-difenil-2- picrilidrazil), conforme a metodologia descrita por Yamaguchi et al. (1998). 0s resultados foram expressos em $\mu \mathrm{M}$ equivalentes de trolox (TE)/g.

As análises estatísticas foram realizadas por análise de variância (one-way ANOVA) e pós-teste de Tukey, utilizando nível de probabilidade inferior a $5 \%(\mathrm{p}<0,05)$.

\section{RESULTADOS E DISCUSSÃ0}

Os resultados da composição nutricional das amostras avaliadas (Figura 1) estão apresentados na Tabela 1 .

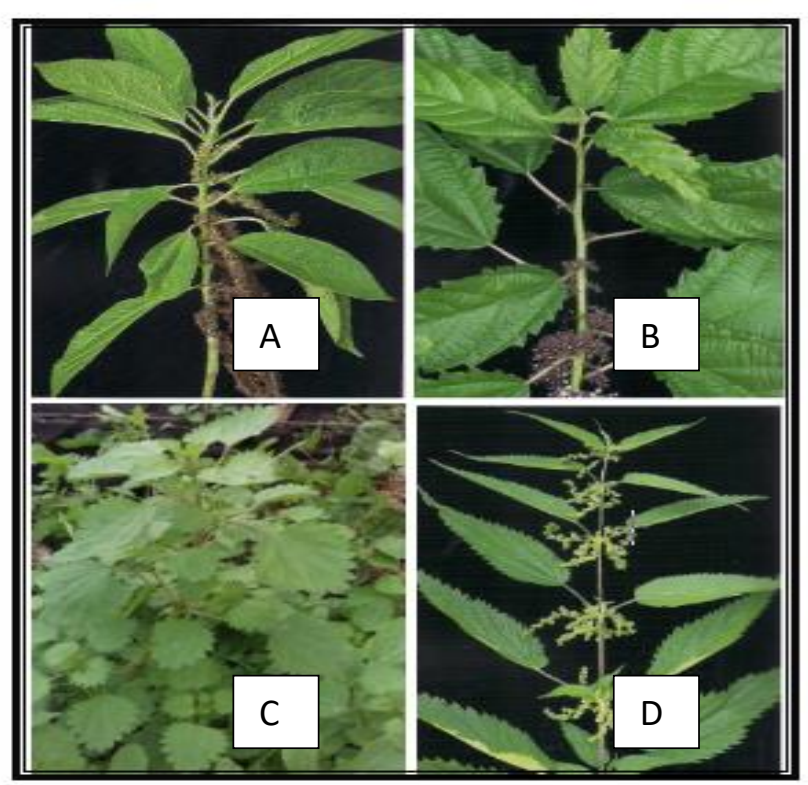

Figura 1. Imagens das espécies B. caudata (A), U. baccifera (B), U. circularis, (C) e U. dioica (D). Fonte: Adaptado de KINUPP; LORENZI, 2014 (A, B e D) e arquivo pessoal dos autores (C). 
Avaliação da Composição Nutricional, Teor Polifenólico e Atividade Antioxidante de Diferentes Espécies da Família Urticaceae

Tabela 1. Composição nutricional (g/100 g) e valor energético das folhas de urtiga.

\begin{tabular}{ccccc}
\hline & U. dioica & B. caudata & U. baccifera & U. circularis \\
\hline Umidade & $8,59 \pm 0,10^{\mathrm{c}}$ & $10,78 \pm 0,03^{\mathrm{b}}$ & $11,02 \pm 0,06^{\mathrm{a}}$ & $10,28 \pm 0,48^{\mathrm{b}}$ \\
Cinzas & $17,27 \pm 0,53^{\mathrm{b}}$ & $10,15 \pm 0,11^{\mathrm{d}}$ & $13,82 \pm 0,16^{\mathrm{c}}$ & $20,95 \pm 0,04^{\mathrm{a}}$ \\
Proteína & $20,88 \pm 0,06^{\mathrm{b}}$ & $21,56 \pm 0,69^{\mathrm{b}}$ & $21,56 \pm 0,00^{\mathrm{b}}$ & $26,60 \pm 0,00^{\mathrm{a}}$ \\
Lipídeos & $5,29 \pm 0,00^{\mathrm{a}}$ & $5,24 \pm 0,24^{\mathrm{ab}}$ & $4,70 \pm 0,27^{\mathrm{b}}$ & $4,53 \pm 0,56^{\mathrm{b}}$ \\
Fibras & $42,67 \pm 0,00^{\mathrm{a}}$ & $43,54 \pm 0,00^{\mathrm{a}}$ & $43,85 \pm 0,00^{\mathrm{a}}$ & $32,28 \pm 0,00^{\mathrm{b}}$ \\
Carboidrato & $6,23 \pm 1,64^{\mathrm{ab}}$ & $7,64 \pm 2,00^{\mathrm{a}}$ & $4,88 \pm 0,51^{\mathrm{bc}}$ & $5,37 \pm 1,18^{\mathrm{b}}$ \\
Energia (Kcal) & $152,35 \pm 1,72^{\mathrm{b}}$ & $168,29 \pm 1,41^{\mathrm{a}}$ & $148,74 \pm 0,07^{\mathrm{c}}$ & $168,59 \pm 0,21^{\mathrm{a}}$ \\
Energia (KJ) & $637,45 \pm 7,18^{\mathrm{b}}$ & $704,13 \pm 5,90^{\mathrm{a}}$ & $622,35 \pm 0,29^{\mathrm{c}}$ & $705,40 \pm 0,89^{\mathrm{a}}$ \\
\hline
\end{tabular}

Fonte: As autoras.

Os valores apresentados na Tabela 1 são resultantes da média de três repetições \pm desvio padrão. Em cada linha, letras iguais não diferem significativamente $(p<0,05)$. Com base nessa Tabela, é possível observar diferenças significativas na composição centesimal em praticamente todos os parâmetros avaliados.

0 teor de umidade obtido nas amostras variou de 8,59 (U. dioica) a 10,78 g/100 g (B. caudata), enquanto a espécie $U$. baccifera apresentou o maior teor de umidade (11,02 g/100 g). Tbatou et al. (2018) descreveram um alto teor de umidade em plantas alimentícias não convencionais de Marrocos in natura, incluindo U. dioica (83 a $95 \mathrm{~g} / 100 \mathrm{~g}$ ). Especificamente para o pó das folhas de Urtiga spp., o teor de umidade relatado é de 7,04 g/ $100 \mathrm{~g}$, inferior ao encontrado no presente estudo (ADHIKARI et al., 2016). As diferenças encontradas nesse parâmetro podem ser explicadas pelo fato de o estudo em questão ter utilizado amostras in natura para a análise.

As técnicas de secagem e moagem, como as empregadas no presente estudo, são alternativas que garantem a estabilidade dos compostos químicos e a manutenção das propriedades biológicas da planta, como previamente observado (UPTON, 2013). Além disso, a obtenção do pó contribui para facilitar e aumentar a produção e o consumo.

0 componente ou o elemento cinzas, apresentou o maior teor em U. circularis $(20,95$ $\mathrm{g} / 100 \mathrm{~g})$, seguido por U. dioica, U. baccifera e B. caudata $(17,27,13,82$ e 10,15 g/100 g, respectivamente). 
ADHIKARI et al. (2016) apresentaram valores de cinzas para Urtica dioica semelhantes aos do presente estudo (16,2 g/100 g). 0 conteúdo de cinzas de um alimento é o resíduo inorgânico, que contempla minerais como cálcio, ferro, manganês, magnésio, entre outros, que permanecem após a queima da matéria orgânica (IAL, 2008). Foi mostrado que esse conteúdo pode variar de acordo com a idade da planta e o corte na colheita, caso esse ocorra antes da floração, como previamente observado (UPTON, 2013). 0 autor descreve, ainda, que os teores de ferro e manganês são maiores nas folhas de plantas jovens, e os de níquel e chumbo são menores em plantas senescentes. Além disso, a presença de cálcio e magnésio é maior nas folhas do que nas raízes (UPTON, 2013).

Em estudo realizado por KINUPP; BARROS (2008) foi demonstrado que a urtiga pode ser considerada uma boa fonte de minerais, como o cálcio $(5300 \mathrm{mg} / 100 \mathrm{~g}$ em Urera aurantiaca), magnésio (1000 mg/100 g em U. circularis) e manganês (263 mg/100 g de U. circularis).

Ao se avaliar o teor de proteína, somente a $U$. circularis apresentou diferença estatisticamente significativa $(26,6 \mathrm{~g} / 100 \mathrm{~g})$, em relação as demais espécies avaliadas. 0s resultados do presente estudo foram semelhantes aos encontrados por KINUPP; BARROS (2008), que observaram diferentes teores de proteína $(19,55$ a $28 \mathrm{~g} / 100 \mathrm{~g})$ em cinco diferentes espécies de Urtiga. Ao se comparar com a espécie Pereskia aculeata (ora-pro-nóbis), uma PANC conhecida pelo seu alto teor de proteína (ROCHA et al., 2008), verifica-se que as urtigas das espécies $U$. baccifera e B. caudata apresentam semelhante teor protéico. Esses achados são relevantes e aumentam as possibilidades de seus usos como fonte de aminoácidos.

0 teor de gordura não apresentou diferença estatística entre as amostras avaliadas e ele ficou de acordo com o reportado por SILVA (2017). É sabido que a época, a região geográfica e o tipo de solo interferem no teor lipídico das espécies de urtiga (SILVA, 2017).

Uma vez que as hortaliças em geral apresentam baixo teor de lipídios, essas podem ser consideradas importantes alternativas para dietas de redução de peso. Além do baixo teor lipídico, também são conhecidas pelo reduzido valor calórico. No presente estudo, observou-se pequena variação no valor calórico total entre as espécies analisadas, com maior teor de carboidratos encontrado na espécie B. caudata $(7,64 \mathrm{~g} / 100 \mathrm{~g})$.

Dentro do grupo dos carboidratos, incluem-se as fibras, constituídas principalmente de polissacarídeos não amido e lignina, sendo resistentes à hidrólise pelas enzimas digestivas humanas (MENEZES; GIUNTINI, 2008). Nesse parâmetro, os teores variaram de 42,67 g/100 g ( $U$. dioica) a 43,85 g/100 g U. baccifera), sem diferença estatística entre as amostras. De acordo com a resolução da Anvisa são considerados alimentos fontes de fibra os que apresentam no mínimo 
$3 \mathrm{~g} / 100 \mathrm{~g}$ e alimentos com alto conteúdo de fibras os que apresentam no mínimo $6 \mathrm{~g} / 100 \mathrm{~g}$ (ANVISA, 2012).

Desse modo, as amostras aqui analisadas podem ser classificadas como alimento com alto conteúdo de fibras. Esses achados são importantes pois as fibras apresentam efeitos fisiológicos benéficos, incluindo a melhora na absorção de nutrientes e redução do colesterol e de glicose séricos (MENEZES; GIUNTINI, 2008).

Além dos macronutrientes, o presente estudo avaliou o conteúdo de fitonutrientes através do conteúdo fenólico total (Tabela 2) e de compostos majoritários (Gráficos 1 - 4). Considerando que esses compostos exercem atividade antioxidante, essa também foi avaliada (Tabela 2).

Os valores apresentados nas tabelas são resultantes da média de três repetições \pm desvio padrão e as letras iguais na mesma coluna indicam que não há diferença estatisticamente significativa.

Tabela 2. Teor de fenólicos totais e atividade antioxidante in vitro.

\begin{tabular}{ccc}
\hline & Fenólicos Totais $(\mathrm{mg} \mathrm{GAE} / 100 \mathrm{~g})$ & DPPH $(\boldsymbol{\mu M}$ TE/g) \\
\hline U. dioica & $260,84 \pm 8,56^{\mathrm{a}}$ & $8,54 \pm 0,12^{\mathrm{a}}$ \\
B. caudata & $274,92 \pm 6,05^{\mathrm{a}}$ & $8,98 \pm 0,05^{\mathrm{a}}$ \\
U. baccifera & $141,18 \pm 1,70^{\mathrm{b}}$ & $5,32 \pm 0,01^{\mathrm{b}}$ \\
U. circularis & $67,90 \pm 1,63^{\mathrm{c}}$ & $3,89 \pm 0,02^{\mathrm{c}}$ \\
\hline
\end{tabular}

Fonte: As autoras.

Ao se avaliar o teor de fenólicos totais, observou-se que os valores encontrados na $B$. caudata e $U$. dioica foram superiores as demais espécies, sendo que a concentração variou de 274,92 a 260,84 mg GAE/100g, respectivamente. Maiores teores de fenólicos podem ser obtidos, no entanto, utilizando diferentes processos extrativos e/ou diferentes solventes. No estudo de MIHAYLOVA et al. (2018), por exemplo, foi encontrado um conteúdo de 143,38 mg GAE/100 g para a tintura feita das folhas de $U$. dioica.

Em outro estudo, MANNION; MENEZES (2010) reportaram $7775 \mathrm{mg}$ GAE/ 100g para o extrato de folhas de $U$. baccifera extraídas com acetato de etila. Resultados semelhantes foram 
descritos por Carvalho et al. (2017), os quais reportaram um conteúdo de $7900 \mathrm{mg} / 100 \mathrm{~g}$ para 0 extrato hidroacetônico de partes aéreas de U. dioica. Embora se consiga atingir maiores teores de compostos fenólicos utilizando solventes orgânicos, a utilização da água como solvente amplia as possibilidades de utilização desses extratos pela área nutracêutica.

Em relação a atividade antioxidante pôde-se observar que a espécie que apresentou maior teor de fenólicos totais foi também a espécie que apresentou a melhor atividade antioxidante (B. caudata). No estudo de MIHAYLOVA et al. (2018) a maior atividade antioxidante foi descrita para $U$. dioica extraída por decocção utilizando água como solvente.

A presença de substâncias antioxidantes em vegetais pode variar largamente em razão da variedade, condições de cultivo, clima e grau de maturação (CAMPOS et al., 2008). Evidências tem mostrado que em condições de fornecimento abundante de nitrogênio, por exemplo, pode haver redução na síntese de compostos fenólicos (G0BBO-NETO; LOPES, 2007).

No presente estudo foram encontrados seis compostos fenólicos (Gráficos 1 - 4, Tabela 3), os quais foram comparados aos padrões, em seus tempos de retenção (Gráfico 5). 0s valores apresentados são resultantes da média de três repetições \pm desvio padrão e utilizou-se a sigla nd para os compostos não detectados.

Pode-se observar que o composto majoritário foi a hesperidina, destacando-se em maior concentração na espécie $U$. baccifera $(116,48 \mu \mathrm{g} / \mathrm{mL})$. Ela foi a única espécie a apresentar canferol e epicatequina em sua composição $(0,65$ e $0,53 \mu \mathrm{g} / \mathrm{mL}$, respectivamente). A concentração de epigalocatequina também foi maior na espécie $U$. baccifera $(6,39 \mu \mathrm{g} / \mathrm{mL})$ e de rutina na B. caudata $(3,05 \mu \mathrm{g} / \mathrm{mL})$. 
Gráfico 1. Identificação de compostos fenólicos em extrato de U. baccifera.

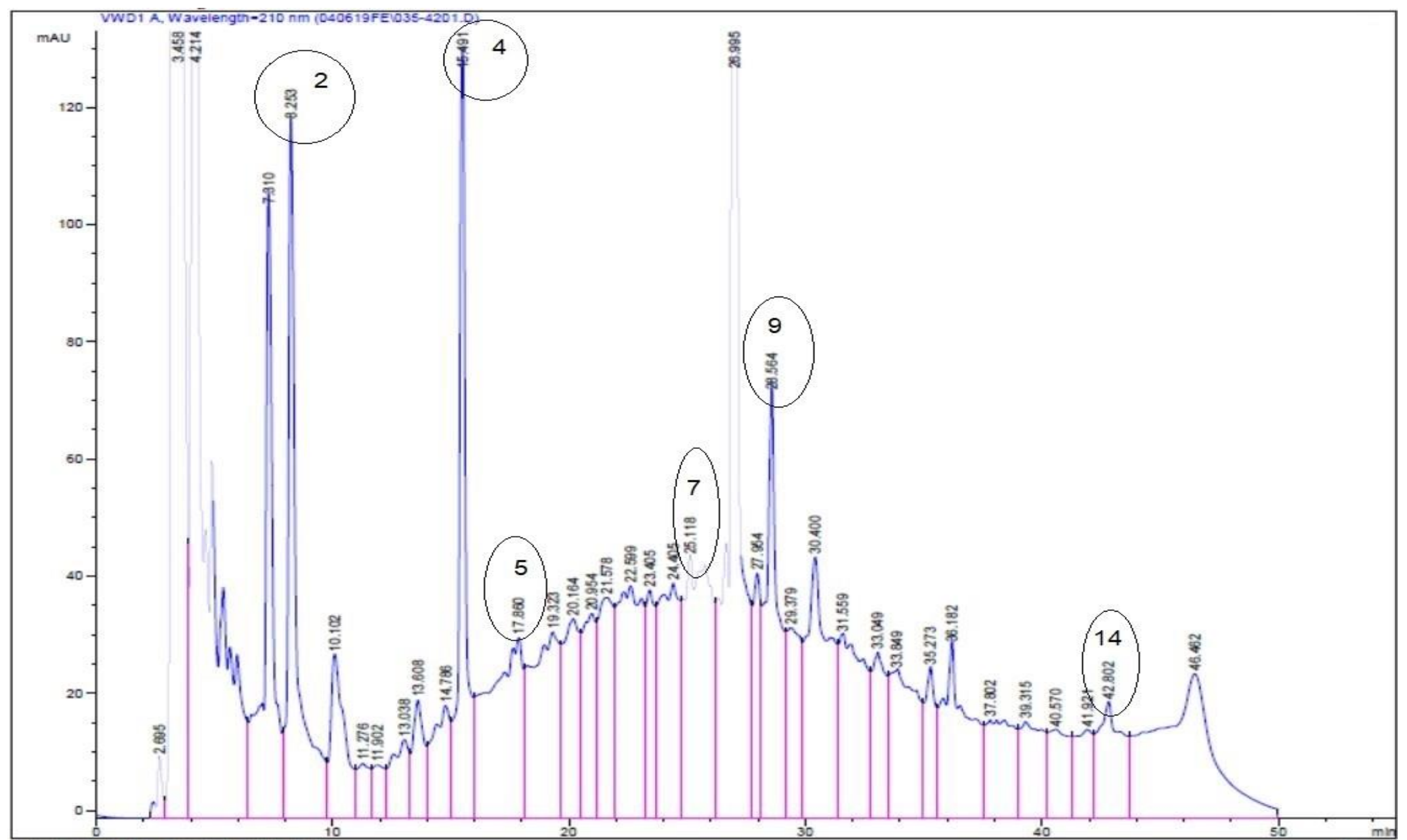

Legenda: 2: epigalocatequina, 4: epicatequina, 5: epigalocatequina galato, 7: rutina, 9: hesperidina e 14: canferol. Fonte: As autoras.

Revista Internacional de Ciências, v. 11, n. 01, p. 243-260, mai-ago, 2021 
Gráfico 2. Identificação de compostos fenólicos em extrato de B. caudata.

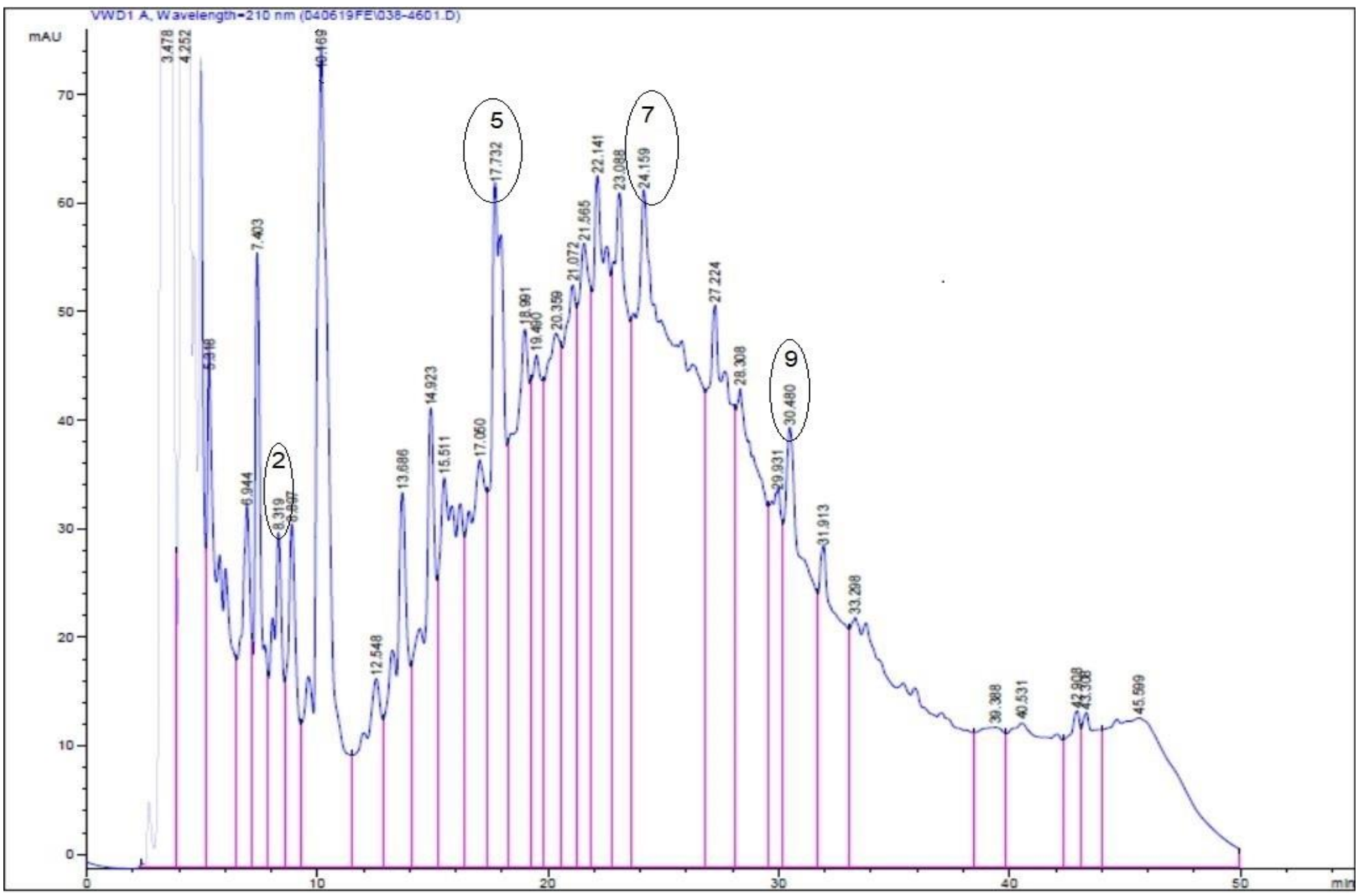

Legenda: 2: epigalocatequina, 5: epigalocatequina galato, 7: rutina e 9: hesperidina. Fonte: As autoras.

Revista Internacional de Ciências, v. 11, n. 01, p. 243-260, mai-ago, 2021 
Gráfico 3. Identificação de compostos fenólicos em extrato de U. dioica.

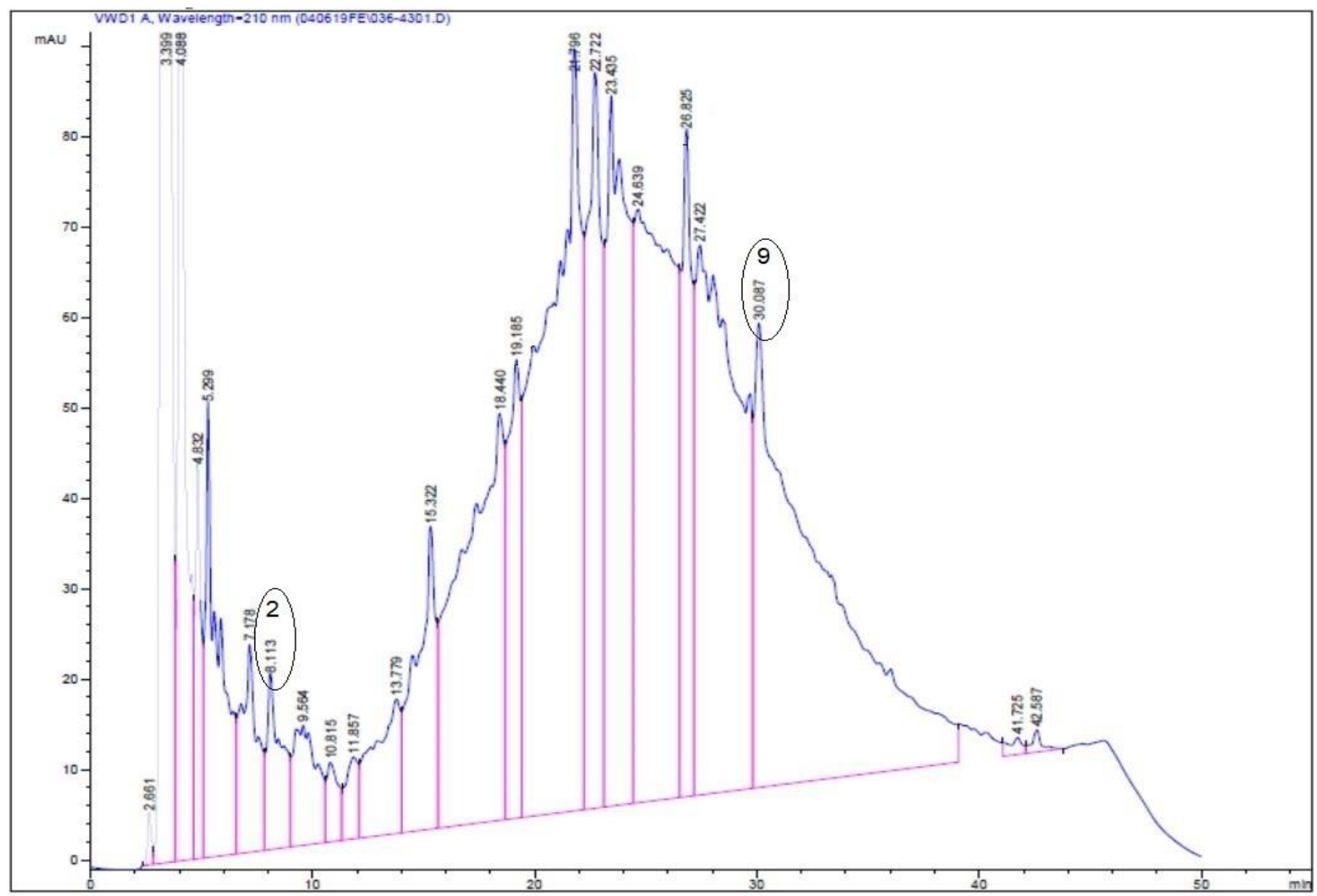

Legenda: 2: epigalocatequina e 9: hesperidina. Fonte: As autoras.

Revista Internacional de Ciências, v. 11, n. 01, p. 243-260, mai-ago, 2021 
Gráfico 4. Identificação de compostos fenólicos em extrato de U. circularis.

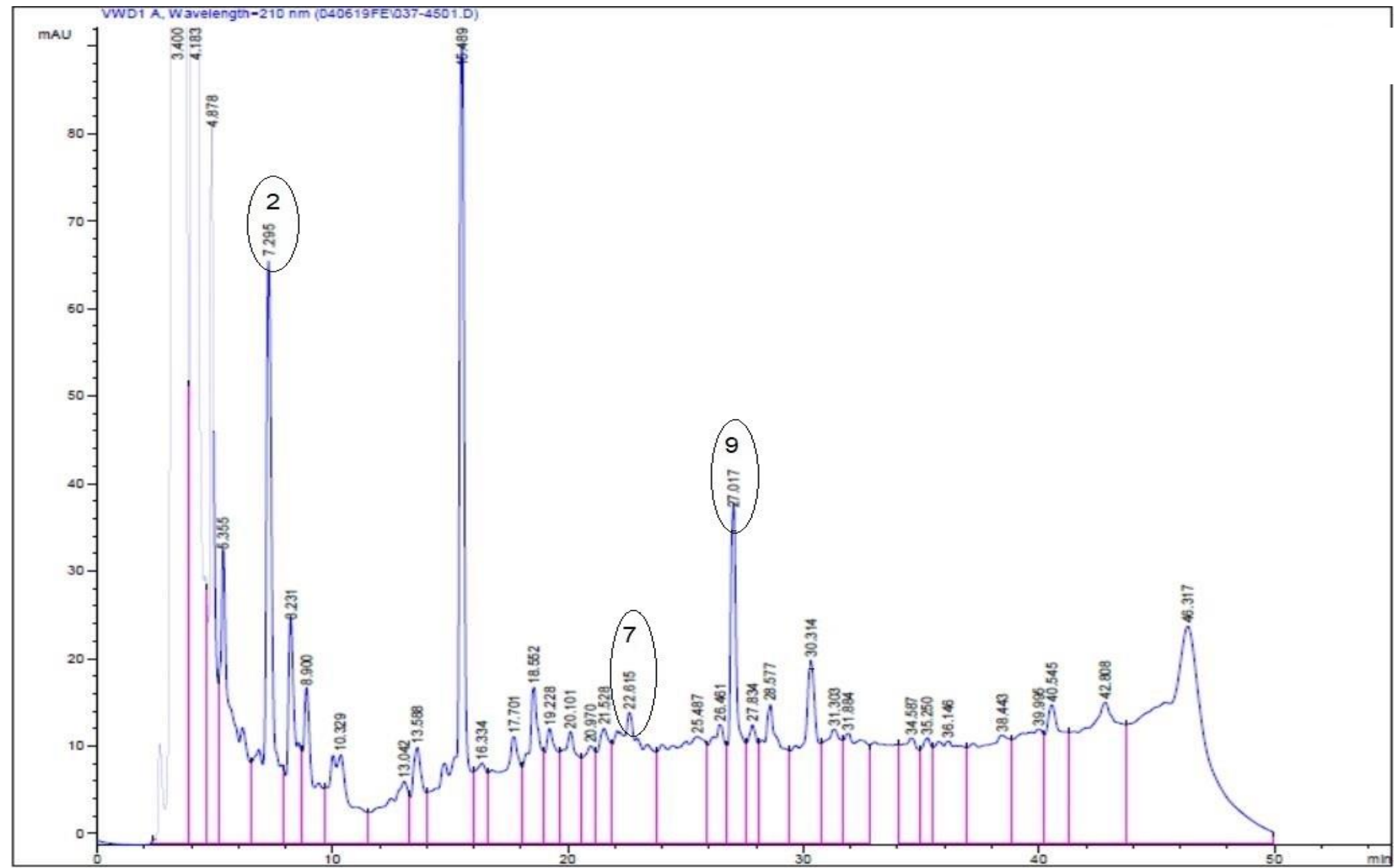

Legenda: 2: epigalocatequina, 7: rutina e 9: hesperidina.

Fonte: As autoras.

Gráfico 5. Perfil cromatográfico dos padrões.

Revista Internacional de Ciências, v. 11, n. 01, p. 243-260, mai-ago, 2021 


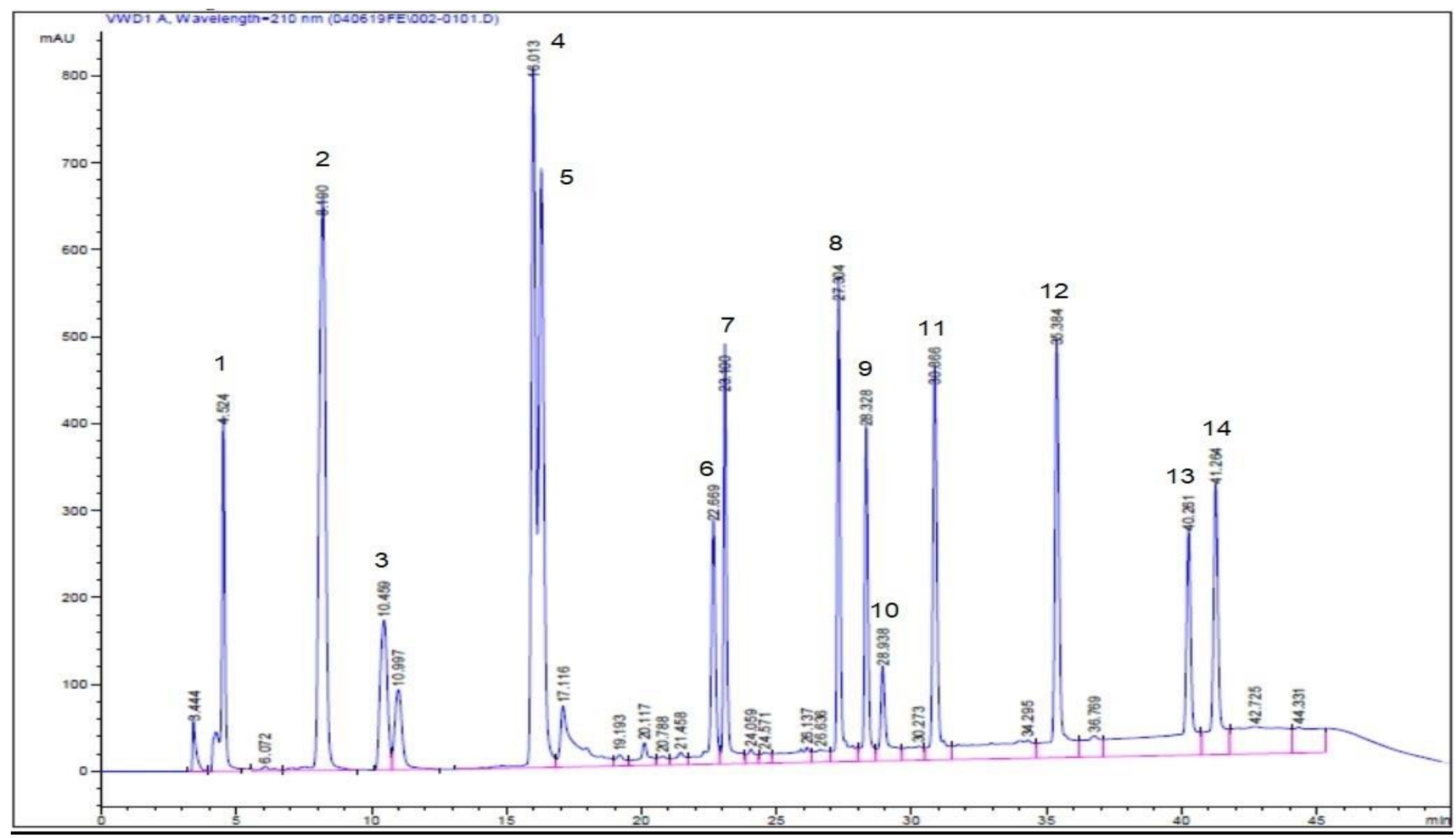

Legenda: 1: ácido gálico, 2: epigalocatequina, 3: catequina, 4: epicatequina, 5: epigalocatequina galato, 6: ácido ferúlico, 7: rutina, 8: naringina, 9: hesperidina, 10: miricetina, 11: resveratrol, 12: quercetina, 13: apigenina e 14: canferol.

Fonte: As autoras.

Revista Internacional de Ciências, v. 11, n. 01, p. 243-260, mai-ago, 2021 
Tabela 3. Concentrações de compostos fenólicos $(\mu \mathrm{g} / \mathrm{mL})$ presente nas espécies de urtiga.

\begin{tabular}{ccccc}
\hline & U. dioica & B. caudata & U. baccifera & U. circularis \\
\hline Epigalocatequina & $0,56 \pm 0,01^{\mathrm{b}}$ & $2,10 \pm 0,01^{\mathrm{a}}$ & $6,39 \pm 0,11^{\mathrm{a}}$ & $3,78 \pm 0,02^{\mathrm{a}}$ \\
Epicatequina & nd & nd & $0,53 \pm 0,01^{\mathrm{a}}$ & nd \\
Epigalocatequina galato & nd & $2,95 \pm 0,00^{\mathrm{a}}$ & $0,79 \pm 0,02^{\mathrm{b}}$ & nd \\
Rutina & nd & $3,05 \pm 0,03^{\mathrm{a}}$ & $2,99 \pm 0,16^{\mathrm{a}}$ & $1,50 \pm 0,07^{\mathrm{b}}$ \\
Hesperidina & $5,45 \pm 0,04^{\mathrm{b}}$ & $2,36 \pm 0,00^{\mathrm{b}}$ & $116,49 \pm 0,94^{\mathrm{a}}$ & $9,79 \pm 0,00^{\mathrm{b}}$ \\
Canferol & nd & nd & $0,65 \pm 0,02^{\mathrm{a}}$ & nd \\
\hline
\end{tabular}

Fonte: As autoras

VAJIC et al. (2018) quantificaram, em extrato da folha da $U$. dioica, rutina $(6,01 \mu \mathrm{g} / \mathrm{mL})$, 2-0-cafeoil ácido málico $(12,29 \mu \mathrm{g} / \mathrm{mL})$ e ácido clorogênico $(15,3 \mu \mathrm{g} / \mathrm{mL})$. Esses compostos apresentaram potencial para reduzir, bem como para eliminar as espécies reativas de oxigênio mais eficientemente do que hidroxitolueno butilato (BHT), um antioxidante comumente empregado como aditivo alimentar. No presente estudo, todavia, a rutina não foi detectada em U. dioica, o que pode ser explicado pela diferente localização geográfica, mas que ainda precisa ser melhor investigado em futuros estudos.

A hesperidina, um glicósideo de flavanona, é um composto fenólico com uma ampla gama de efeitos biológicos. Estudos crescentes apontam 0 seu efeito benéfico contra 0 desenvolvimento de doenças neurodegenerativas como demência e Alzheimer (HAJIALYANI et al., 2019; KIM et al., 2019).

Além disso, a hesperidina demonstrou ser eficaz contra a dor neuropática diabética, e, em conjunto com a insulina, foi eficiente no controle da hiperglicemia e hiperlipidemia, que desregulam a produção de espécies reativas e liberam citocinas pró-inflamatórias (HAJIALYANI et al., 2019). Segundo XIONG et al. (2019), a sua ação sobre o metabolismo lipídico e glicêmico se dá através da mediação das vias de sinalização AMPK, PPAR e de NF- $\kappa \mathrm{B}$, por meio das atividades atividade antioxidante, anti-apoptótica e anti-inflamatória exibida por esse flavonoide.

Com essa gama de efeitos protetores que os compostos fenólicos exibem há possibilidades de explorá-los na elaboração de diferentes produtos. Um exemplo nesse sentido é a incorporação desses extratos em alimentos. Recentemente foi mostrado que biscoitos amanteigados enriquecidos com farinha de extrato de $U$. dioica exibiram redução da oxidação lipídica, com consequente aumento da estabilidade da cor durante o armazenamento 
(KOZLOWSKA et al., 2019). Dessa forma, abrem-se novas possibilidades de estudos e aplicações envolvendo os extratos de urtiga.

\section{CONCLUSÃO}

A família Urticaceae possui uma ampla adaptação a diferentes ambientes, podendo variar sua composição nutricional e fenólica. Inicialmente conhecidas pela presença de tricomas urticantes em suas folhas, essas plantas agora passam a ser reconhecidas também como PANC's, e aos poucos vem ganhando espaço em preparações alimentícias. Este estudo mostrou, pela primeira vez, que quatro espécies comestíveis de urtiga possuem rica composição centesimal, destacando-se por seu conteúdo de minerais e fibras. Além disso, exibem significativa capacidade antioxidante e teor de compostos fenólicos totais, destacando-se a hesperidina, um flavonoide com importante atividade farmacológica. Por fim, por ser um alimento ainda pouco estudado, esses achados poderão servir de base para futuros estudos nas áreas nutracêutica e farmacêutica para o desenvolvimento de produtos com potencial tecnológico.

\section{AGRADECIMENTOS}

Os autores agradecem à Universidade de Caxias do Sul. Michele Nascimento participou das etapas de coleta, preparo e análises das amostras, interpretação dos dados, redação e revisão final. Luana Minello e Elizete Maria Pesamosca Facco participaram das análises e interpretações de dados e da revisão final. Catia Santos Branco participou das análises estatísticas, interpretação dos dados, edição e revisão final. Valdirene Camatti Sartori participou da concepção e desenho do estudo, análises e interpretações de dados e revisão final. Gabriela Chilanti participou da concepção e desenho do estudo, análises e interpretações de dados e revisão final.

\section{REFERÊNCIAS BIBLIOGRÁFICAS}

ADHIKARI, B. M.; BAJRACHARYA, A.; SHRESTHA, A.K. Comparison of nutritional properties of stinging nettle (Urtica dioica) flour with wheat and barley flours. Food Science \& Nutrition, 4(1):119-124, 2016.

AGÊNCIA NACIONAL DE VIGILÂNCIA SANITÁRIA (ANVISA). Resolução da diretoria colegiada - RDC $n^{0} 54$, de 12 de novembro $\quad$ de $2012 . \quad$ Disponível em: http://portal.anvisa.gov.br/documents/\%2033880/2568070/rdc0054_12_11_2012.pdf/c5ac23fd-974e-4f2c-9fbc48f7e0a31864. Acesso em 03 março 2020. 
ALMEIDA, M.E.F.; CORRÊA, A.D. Utilização de cactáceas do gênero Pereskia na alimentação humana em um município de Minas Gerais. Revista Ciência Rural, 42(4):752-756, 2012.

A0AC. Official methods of analysis of the Association of Official Analytical Chemists. 18th ed. Washington: A0AC, 2011.

CAMPOS, F.M.; MARTINO, H.S.D.; SABARENSE, C.M.; PINHEIR0-SANT'ANA, H.M. Estabilidade de compostos antioxidantes em hortaliças processadas: uma revisão. Alimentos e Nutrição Araraquara, 19(4):481-490, 2008.

CARVALHO, A.R.; COSTA, G.; FIGUEIRINHA, A.; LIBERAL, J.; PRIOR, J.A.V.; LOPES, M.C.; CRUZ, M.T.; BATISTA, M.T. Urtica spp.: Phenolic composition, safety, antioxidant and anti-inflammatory activities, Food Research International, 99(1): 485-494, 2017.

CASEMIR0, I.P.; VENDRAMIN, A.L.A.; Plantas alimentícias não convencionais no Brasil: o que a Nutrição sabe sobre esse tema? DEMETRA Alimentação, Nutrição \& Saúde, DOI: 10.12957/demetra.2020.42725.

DEL RIO, D.; RODRIGUEZ-MATEOS, A.; SPENCER, J.P.E.; TOGNOLINI, M.; BORGES, G.; CROZIER, A. Dietary (poly)phenolics in human health: structures, bioavailability, and evidence of protective effects against chronic diseases. Antioxidants \& Redox Signaling, 18(14): 1818-92, 2013.

DHOUIBI, R.; AFFES, H.; SALEM, M.B.; HAMMAMI, S.; SAHNOUN, Z.; ZEGHAL, K.M.; KSOUDA, K. Screening of pharmacological uses of Urtica dioica and others benefits. Progress in Biophysics and Molecular Biology, 150: 6777, 2020,

G0BBO-NETO, L.B.; LOPES, N.P. Plantas medicinais: fatores de influência no conteúdo de metabólitos secundários. Química Nova, 30: 374-381, 2007.

HAJIALYANI, M.; FARZAEI, M. H.; ECHEVERRÍA, J.; NABAVI, S.; URIARTE, E.; SOBARZO-SÁNCHEZ, E. Hesperidin as a neuroprotective agent: a review of animal and clinical evidence: a review of animal and clinical evidence. Molecules, 24(3): 648, 2019.

INSTITUT0 ADOLF0 LUTZ (IAL). Método físico-químicos para análise de alimentos. São Paulo: Instituto Adolfo Lutz, 1020 p, 2008.

KIM, J.; WIE, M.B.; AHN, M.; TANAKA, A.; MATSUDA, H.; SHIN, T. Benefits of hesperidin in central nervous system disorders: a review. Anatomy \& Cell Biology, 52(4):369-377, 2019.

KINUPP, V.F.; BARROS, I.B.I. Riqueza de plantas alimentícias não convencionais na região metropolitana de Porto Alegre, Rio Grande do Sul. Revista Brasileira de Biociências, 5:1-3, 2007.

KINUPP, V.F.; BARROS, I.B.I. Teores de proteína e minerais de espécies nativas, potenciais hortaliças e frutas. Ciência e Tecnologia de Alimentos, 28(4): 846-857, 2008.

KINUPP, V.F.; LORENZI, H. Plantas alimentícias não convencionais (PANCs) no Brasil: guia de identificação, aspectos nutricionais e receitas ilustradas. Instituto Plantarum de Estudos da Flora São Paulo: Plantarum, 2014, 768 p.

KOZLOWSKA, M. Herbal extracts incorporated into shortbread cookies: impact on color and fat quality of the cookies. Biomoléculas, 9(12): 2-16, 11, 2019.

KREGIEL, D.; PAWLIKOWSKA, E.; ANTOLAK, H. Urtica spp.: Ordinary plants with extraordinary properties. Molecules, 23(7): 1664, 2018.

LEAL, M. L. Knowledge, use, and disuse of unconventional food plants. Journal of Ethnobiology And Ethnomedicine, 14: 2-9, 2018.

MANNION, F.; MENEZES, F. S. Antioxidant activity of Urera baccifera Gaud extracts. The Trinity College Dublin Journal of Pharmacy \& Pharmaceutical Sciences, 2: 8-9, 2010. 
MENEZES, E.W; GIUNTINI, E.B. Fibra alimentar. In: Philippi ST, editor. Pirâmide dos alimentos: fundamentos básicos da nutrição. São Paulo: Manole, 2008. pp. 249-63.

MIHAYLOVA, D.; VRANCHEVA, R.; DESSEVA, I.; IVANOV, I.; DINCHEVA, I.; POPOVA, M.; POPOVA, A. Analysis of the GC-MS of volatile compounds and the phytochemical profile and antioxidant activities of some Bulgarian medicinal plants. Zeitschrift für Naturforschung C, 74(1-2): 2-10, 2018

MORELLI, L.L.L. Avaliação de compostos fenólicos majoritários em geleia de uva produzida com a variedade IAC138-22 (máximo). 2011. 133 p. Dissertação (Mestrado) - Universidade Estadual de Campinas, Campinas, SP.

OLIVEIRA, V.F.G.; NASCIMENTO, R.S.; OLIVEIRA, S.C.; SÁ LEITÃO, C.S. Plantas alimentícias não convencionais usadas na gastronomia. Revista de Produção Acadêmico-Científica; 6-15, 2016.

PEDERNEIRAS, L.C.; COSTA, A.F.; ARAÚJJ, D.S.S; CARAUTA, J.P.P. Ulmaceae, cannabaceae e urticaceae das restingas do estado do Rio de Janeiro. Rodriguésia, 62(2): 300-313, 2011.

ROCHA, D.R.C.; PEREIRA JÚNIOR, G.A.; VIEIRA, G.; PANTOJA, L.; SANTOS, A.S.; PINTO, N.A.V.D. Macarrão adicionado de ora-pro-nóbis (Pereskia Aculeata Miller) desidratado. Alimentos e Nutrição Araraquara, 19(4):459-465, 2008.

SARTORI, V.C.; THEODOR0, H.; MINELLO, L.V.; PANSERA, M.R.; BASSO, A.; SCUR, L. Plantas Alimentícias Não Convencionais - PANC: resgatando a soberania alimentar e nutricional. Caxias do Sul, RS: Educs, 2020. 118 p.

SILVA, J.0. Caracterização nutricional e atividade biológica de urtiga selvagem (Urtica dioica L.). 2017.95 f. Dissertação (Mestrado em Qualidade e Segurança Alimentar) - Escola Superior Agrária de Bragança, Bragança.

SINGLETON, V.L.; ROSSI, J.A. Colorimetry of total phenolics with phosphomolybdic-phosphotungstic acid reagents. American Journal of Enolology and Viticulture, 16(3):144-158, 1965.

SOUZA, M.O.; BRANCO, C.S.; SENE, J.; DALLAGNOL, R.; AGOSTINI, F.; MOURA, S.; SALVADOR, M. Antioxidant and antigenotoxic activities of the brazilian pine Araucaria angustifolia (Bert.) 0. Kuntze. Antioxidants, 3: 24-37, 2014.

TBATOU, M.; KABIL, M.; BELAHYAN, A.; BELAHSEN, R. Dietary potential of some forgotten wild leafy vegetables from Morocco. International Food Research Journal, 25(5):1829-1836, 2018.

UPTON, R. Stinging nettles leaf (Urtica dioica L.): extraordinary vegetable medicine. Journal of Herbal Medicine, 3:9-38, 2013.

VAJIC, U.J.; GUJIC-MILANOVIC, J.; MILORADOVIC, Z.; JOVOVIC, D.; IVANOV, M.; DANIJELA, K.; SAVIKIN, K.; BUGARSKI, B.; MIHAILOVIC-STANOJEVIC, N. Urtica dioica L. leaf extract modulates blood pressure and oxidative stress in spontaneously hypertensive rats. Phytomedicine, 15; 46:39-45, 2018.

XIONG, H.; WANG, J.; RAN, Q.; LOU, G.; PENG, C.; GAN, Q.; HU, J.; SUN, J.; YA0, R.; HUANG, Q. Hesperidin: a therapeutic agent for obesity. Drug Design, Development and Therapy, 13: 3855-3866, 2019.

YAMAGUCHI, T.; TAKAMURA, H.; MATOBA, T.; TERA0, J. HPLC Method for Evaluation of the free radical scavenging activity of foods by using 1,1-Diphenyl-2- picrylhydrazyl. Bioscience, Biotechnology and Biochemistry, 62(6): 12011204, 1998. 